speculatively interpreted to mean that both putative transporter genes are essential for peptide supply to class I molecules and that "peptide supply factor" is in fact a heterodimer of the two gene products (see figure). Resolution of this question should help the search for a direct demonstration that the putative transporter proteins do actually pump peptides.

Peter Parham is in the Departments of Cell Biology, and Microbiology and Immunology, Stanford University School of Medicine, Stanford, California 94305, USA.

\title{
The answer is near
}

\section{Charles D. Dermer}

Debate has continued over the distances to the sources of brief, intense flashes of $\gamma$ radiation called $\gamma$-ray bursts since their discovery was announced ${ }^{1}$. The apparently isotropic distribution of burst directions on the sky has made it impossible to determine whether the bursts arise in our Galaxy or have an extragalactic or cosmological origin. Attempts to identify steady counterparts at other wavelengths have also been fruitless, except in the case of the unusual 5 March 1979 event which, if located in the Large Magellanic Cloud, would place 'normal' bursts far outside the Galaxy. On page 296 of this issue ${ }^{2}$, a French-Soviet collaboration concludes, from an analysis of the statistical properties of burst data, that $\gamma$-ray bursters are nearby galactic phenomena. If this conclusion is substantiated, an exciting chapter in highenergy astrophysics will finally have come to an end.

Gamma-ray bursts are cosmic fireworks that flare up for a few seconds, emit the bulk of their energy in $\gamma$-ray photons, and then become invisible at all wavelengths, in most cases never to recur. Analyses of the spatial distribution of bursts are therefore necessarily statistical, involving the brightness time histories, positions on the sky and bursting rates. Because of the great variations in the brightness histories, these are usually replaced by a single value representing the burst strength, normally chosen to be peak flux or peak detector count rate.

The technique used by Atteia and colleagues ${ }^{2}$, and some of the pitfalls such studies encounter, can be illustrated through the more familiar example of star counts in the night sky. The figure shows the visual magnitude distribution of all stars in the sky, as well as the magnitude distribution of stars in the direction of the galactic plane and pole $^{3}$. (Magnitude is a logarithmic measure of intrinsic brightness, the faintest stars having the largest magnitude.) Slopes of isotropic and disk distributions of equalbrightness stars are shown for comparison. At magnitudes $m<2$, the plots become limited by statistics: there are not enough stars brighter than second magnitude to conclude anything about their distribution. The all-sky distribution is roughly isotropic for $m<5$, and corresponds to a disk distribution for $5 \leq m \leq 15$. At fainter magnitudes we are evidently seeing past the edge of the disk, where the star density becomes small.
The shape of the stellar distribution function depends on whether we are looking toward the galactic pole or plane. In the polar direction, we start to run out of stars with $m$ $>10$, whereas the edge of the disk distribution in the direction toward the galactic plane occurs for much fainter stars with $m>15$. Given a representative source whose distance we know, such as the Sun, a reasonably accurate picture of the Galaxy as a flattened disk distribution, with vertical scale height of about 100 parsecs, can be constructed.

The approach by Atteia et al. is similar, but instead of magnitude, they use the variable $V / V_{\max }$ introduced by Schmidt ${ }^{4}$ in his studies

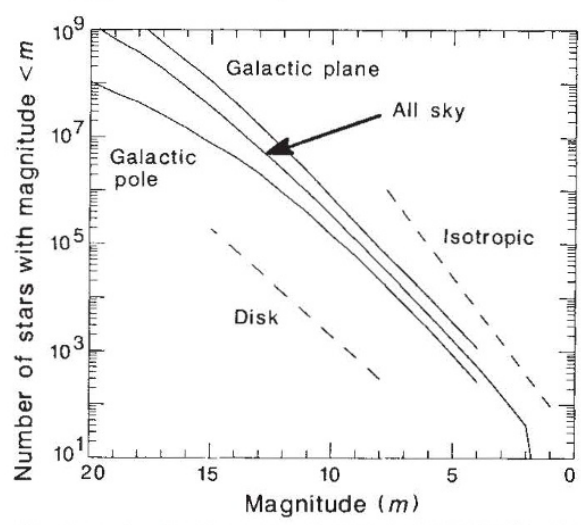

Magnitude distribution of stars observed in all directions (all sky), and in the direction of the galactic plane and pole. The density of stars toward the galactic plane and pole are scaled to the all-sky density and total number distribution.

of quasars. Here $V$ stands for volume, and corrects the measured peak count rate for the increase, proportional to $r^{3}$, in the number of sources at distance $r$ and for the $1 / r^{2}$ decrease in their apparent flux; $V_{\max }$ is the maximum volume that can be probed by a detector because of threshold limitations. The average of a sample of $V / V_{\max }$ values equals 0.5 if $\gamma$-ray bursts are uniformly distributed in space, and all detector biases are taken into account. In terms of the figure, the $V / V_{\max }$ test provides a measure of the deviation of a distribution of stars brighter than a limiting magnitude from one with slope 0.6 , which is the slope of the isotropic distribution.

Normally the $V / V_{\max }$ test is made without reference to direction. The new approach in the French-Soviet study is to consider the directional properties of faint bursts only, that is, those with large $V / V_{\max }$ values which presumably originate at large distances. By selecting only these faint distant bursts (or, referring again to the figure, only dim stars), the spatial anisotropies are magnified. The authors find statistically significant evidence for the dim distant bursts to lie in the galactic plane. Such a result, if true, goes a long way toward demolishing all extragalactic models of $\gamma$-ray bursts.

The approach seems sound: although the $\gamma$-ray bursts will have a broad range of luminosities, rather than the single 'standard candle' luminosity implicit in the analysis, it seems unlikely that the difference would alter the conclusions. And although obscuration by dust and gas can distort optical surveys, the effect would be negligible for $\gamma$ rays, and in any case would be most marked for bursts in the plane of the Galaxy. In focusing on the peak count rates from bursts, the authors of the study have chosen the measure of intensity shown ${ }^{5,6}$ to be most free from instrumental bias. The authors show that it is highly unlikely that the excess of bursts seen in the plane is a result of chance; although the analysis involves the lowintensity 'cut off' value of $V / V_{\text {max }}$, one presumes that the conclusion does not depend on the particular dividing line between 'bright' and 'faint' bursts. Also, one might ask whether the enhancement in the galactic plane would have been seen if their approach had been applied to other catalogues of bursts, in particular the Konus catalogue with over 60 different localized bursts $^{7-10}$.

Although there will always be sceptics in a subject as fraught with detector biases and statistical uncertainties as $\gamma$-ray astronomy, this work presents the strongest case to date that $\gamma$-ray bursters are galactic. Compelling evidence that the burst distribution is spatially limited has been known since the balloon test ${ }^{11}$ of the prototype of the burst detector now flying on the Gamma Ray Observatory, so these results are by no means unexpected. The burst experiment on GRO is now recording $\gamma$-ray bursts at a frequency of about 1 per day (G. J. Fishman, personal communication), so confirmation or refutation of this study is not, perhaps like the bursters themselves, far away.

Charles D. Dermer is in the Department of Space Physics and Astronomy, Rice University, Houston, Texas 77251-1892, USA.

1. Kiebesadel, R. W., Strong, I. B. \& Olson, R. A. Astrophys. J. 182, L85-L88 (1973).

2. Atteia, J. L. et al. Nature 351, 296-298 (1991)

3. Allen, C. W. Astrophysical Quantities 3rd edn p. 244 (Athlone. London, 1976).

4. Schmidt, M. Astrophys. J. 151, 393-409 (1968).

5. Mazets, E. P. in 19th int. Cosmic Ray Conf. (La Jolla) 9. 415-430 (1986).

6. Paczyński, B. \& Long, K. Astrophys. J. 333, 694-699 (1988).

7. Mazets, E. et al. Astrophys. Space Sci. 80, 3-83 (1981) 8. Mazets, E. et al. Astrophys. Space Sci. 80. 85-117 (1981). 9. Atteia, J. et al. Astrophys. J. Suppl. 64, 305-382 (1987). 10. Higdon, J. C. \& Schmidt, M. Astrophys. J. 355, 13-17 (1990).

11. Meegan, C. A., Fishman, G. J. \& Wilson, R. B. Astrophys. J. 291, 479-485 (1985). 